\title{
Developmental enamel defects in tuberous sclerosis: a clinical genetic marker?
}

\author{
N Flanagan, W J O'Connor, B McCartan, S Miller, J McMenamin, R Watson
}

Department of

Dermatology, St

James's Hospital,

James's Street, Dublin,

Ireland

N Flanagan

W J O'Connor

R Watson

Department of Oral Surgery, Oral

Medicine and Oral

Pathology, Dublin

Dental Hospital,

Dublin, Ireland

B McCartan

Department of Radiology, St James's

Hospital, James's

Street, Dublin, Ireland

$S$ Miller

\section{Department of Neurology, Our Lady's \\ Hospital for Sick \\ Children, Crumlin, \\ Dublin, Ireland \\ J McMenamin}

Correspondence to: Dr Flanagan, Department of Dermatology, Royal Victoria Infirmary, Newcastle upon

Tyne NE1 4LP, UK.

Received 21 June 1996 Revised version accepted for publication 8 April 1997

\begin{abstract}
Ten probands with tuberous sclerosis (TS) and 20 first degree relatives were examined for evidence of pitted enamel hypoplasia; $100 \%$ of TS patients had pitting, compared to $65 \%$ of relatives and $72 \%$ of 25 controls. We found that $70 \%$ of TS cases had more than 14 pits per person compared with only $5 \%$ of relatives and $4 \%$ of controls; $85 \%$ of relatives and $84 \%$ of controls had fewer than six pits per person. Our results confirm that significantly increased numbers of dental enamel pits are found in persons with TS compared to controls. These results suggest that examination for the presence or absence of dental enamel pits is not a useful screening test for first degree relatives to detect otherwise unsuspected subjects with tuberous sclerosis. However, the lack of pits in first degree relatives in our study is probably largely because none of the relatives appeared to carry the TS gene. $(\mathcal{M}$ Med Genet 1997;34:637-639)
\end{abstract}

Keywords : tuberous sclerosis; teeth; dental pits

Tuberous sclerosis (TS) is an autosomal dominant disorder characterised by hamartoma formation in many organs. The prevalence of the disease is about 1 per 10000 . Genetic linkage analysis studies have indicated that approximately half of all TS families show linkage to chromosome $9 \mathrm{q} 34$ and half to chromosome 16p13. Positional cloning efforts are now under way in several centres to attempt to find the TSC1 gene. The TSC2 gene on chromosome 16 has recently been isolated. ${ }^{1}$ Seventy percent of cases appear to arise as new mutations, but this rate may be falsely high as the manifestations of the disease may be subtle. There are a few families with documented non-penetrance. ${ }^{23}$ Several families have been described in which two affected children were born to parents who appeared to be completely normal after thorough evaluation. The more clinical or special tests which are carried out on apparently unaffected first degree relatives, the more likely that some abnormality will be detected. Previous studies have established that dental enamel pitting is common in TS. ${ }^{4-8}$ This study was performed to assess the value of dental examination as a screening test for first degree relatives of apparent sporadic TS cases.

\section{Patients and methods}

Thirty-one subjects from 11 families of TS patients participated, comprising $11 \mathrm{TS}$ cases,
Table 1 Prevalence of dental pitting in TS probands compared with relatives and controls

\begin{tabular}{llll}
\hline & No of patients & \% with pits & Mean pits per tooth \\
\hline Probands & 10 & 100 & 1.27 \\
Relatives & 20 & 65 & $0.30^{\star}$ \\
Controls & 25 & 72 & $0.24^{\star \star}$ \\
\hline
\end{tabular}

$\star$ Probands $v$ relatives, $\mathrm{p}=0.0005, \mathrm{IRR}=2.3$.

$\star \star$ Probands $v$ controls, $\mathrm{p}<0.0005, \mathrm{IRR}=2.7$

16 parents, and four sibs. All probands fulfilled the diagnostic criteria for definite TS and none of the relatives had any features to suggest a diagnosis of TS. ${ }^{9}$ Ten TS patients underwent dental examination, the remaining child being uncooperative. Both parents were available for examination in seven families. Six parents were not examined because they were unavailable for examination, edentulous, or had died. The median age of the TS cases was 24 years (range 5-37) and of the first degree relatives 41 years (range 8-65). Twenty-five controls, median age 23 years (range 21-31), all of whom were young adult dentists, dental students, or dental nurses, also participated in the dental part of the study. Any teeth which had been restored or crowned were excluded. Permanent and deciduous teeth were examined. TS patients and their first degree relatives also underwent full skin examination (including Wood's light examination) and renal ultrasound.

The technique used in the dental examination was modified from that of Mlynarczyk. ${ }^{5}$ All dental examinations were carried out by the same registered dental hygienist using a dental chair, dental light, and a sharp dental explorer. The labial surfaces of the 12 anterior teeth (incisors and canines) were given a standard dental prophylaxis, following which a disclosing solution (stain) was applied using a sponge. The teeth were dried using a cotton roll. The teeth were explored carefully with the probe

Table 2 Mean numbers of pits, and numbers of teeth, for each tooth type examined in TS cases, first degree relatives, and controls

\begin{tabular}{|c|c|c|c|c|c|c|}
\hline & \multicolumn{2}{|c|}{ Probands } & \multicolumn{2}{|c|}{ Relatives } & \multicolumn{2}{|c|}{ Controls } \\
\hline & Mean & No & Mean & No & Mean & No \\
\hline \multicolumn{7}{|l|}{ Maxilla } \\
\hline $\begin{array}{l}\text { Central } \\
\text { incisor }\end{array}$ & 1.95 & 19 & 0.67 & 33 & 0.22 & 50 \\
\hline $\begin{array}{l}\text { Lateral } \\
\text { incisor }\end{array}$ & 0.80 & 20 & 0.26 & 31 & 0.62 & 50 \\
\hline $\begin{array}{r}\text { Canine } \\
\text { Mandible }\end{array}$ & 1.40 & 20 & 0.46 & 35 & 0.40 & 50 \\
\hline $\begin{array}{l}\text { Central } \\
\text { incisor }\end{array}$ & 0.85 & 20 & 0.08 & 38 & 0.02 & 50 \\
\hline $\begin{array}{l}\text { Lateral } \\
\text { incisor }\end{array}$ & 1.00 & 20 & 0.11 & 38 & 0.08 & 50 \\
\hline Canine & 1.72 & 20 & 0.29 & 38 & 0.12 & 50 \\
\hline
\end{tabular}




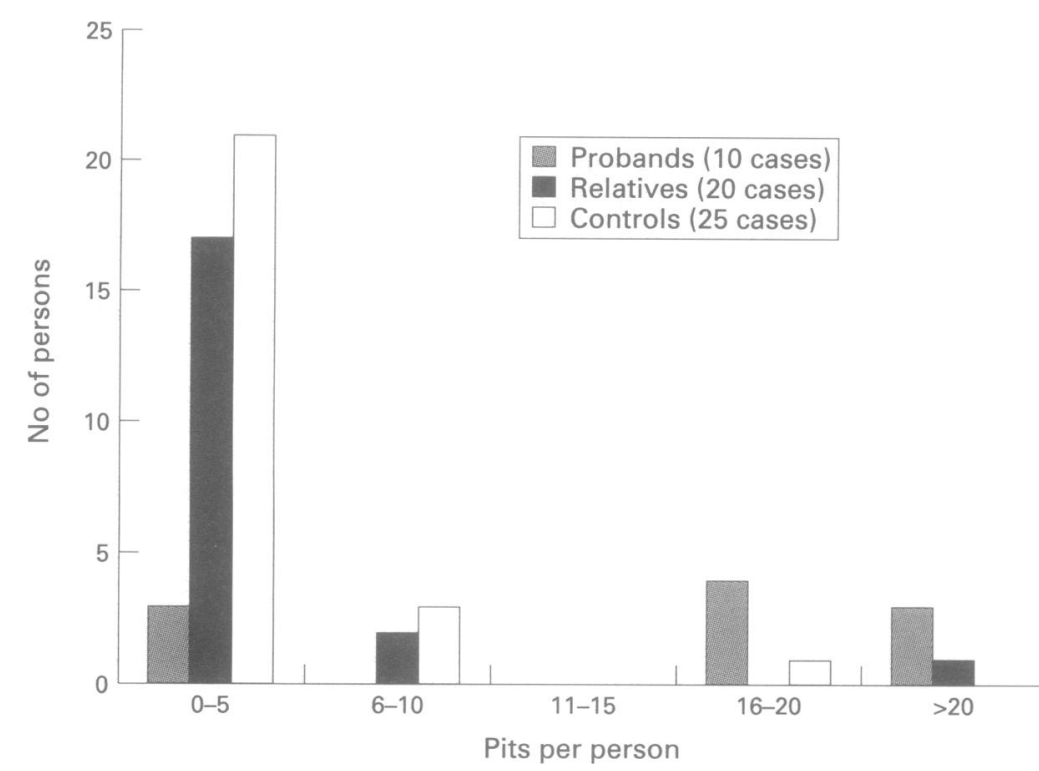

Figure 1 Number of pits per person in probands, first degree relatives, and controls.

using light pressure. Pits were detected visually and confirmed if they would "catch and keep" the probe from moving across the surface of the tooth. Each examination (excluding prophylaxis and staining) took approximately five minutes. The first 30 examinations were observed by one of the authors, a dentist, to ensure standardisation.

Statistical analysis was carried out using Poisson regression and results were expressed as (incidence) relative risks (IRR).

\section{Results}

All 10 TS patients showed pitting (mean 1.27 per tooth) compared with $65 \%$ of first degree relatives (13 of 20 , mean 0.30 per tooth) and $72 \%$ of controls (18 of 25 , mean 0.24 per tooth) (table 1). Although pits were common in controls, the majority ( $84 \%$ ) had fewer than six pits, whereas $70 \%$ of probands had more than 15 pits (fig 1). TS patients had an increased number of pits for each tooth type compared to controls and relatives (table 2). One parent was found to have 25 pits and one control had 16 pits. Cutaneous examination of both and renal ultrasound of the parent showed no other features of TS. Some pits were relatively large and easily visible to the naked eye while other, smaller pits were detectable only with staining and probing. Both large, visible pits and small pits were detectable in all three groups.

Results were analysed by status (case, relative, or control) and by tooth type (maxillary central incisor, etc). TS patients were found to have significantly increased numbers of dental enamel pits when compared with controls (IRR=2.7, p<0.0005) and when compared with relatives (IRR $=2.3, p=0.0005)$. There was no significant difference in the overall number of dental enamel pits between relatives and controls (IRR $=1.1, \mathrm{p}=0.45)$.

Six of the nine TS patients $(66 \%)$ who underwent renal ultrasound showed multiple bilateral angiomyolipomata and this finding was not seen in any of the 20 first degree relatives. All but one proband showed cutaneous manifestations of TS and none of the first degree relatives had any cutaneous manifestations of TS.

\section{Discussion}

Pits were first described by Hoff $e t a l^{4}$ in 1975 in a series of six patients with TS. Since then several studies have shown that dental enamel pits are a common finding in $\mathrm{TS}^{4-8}$ (table 3 ). Roach et $a l^{9}$ included dental enamel pits as a tertiary feature in the diagnostic criteria for TS. Our finding of $100 \%$ prevalence of dental enamel pits in probands correlates with the study by Mlynarczyk. ${ }^{5}$ Thus dental pits may be the commonest manifestation of TS and therefore a helpful marker in the diagnosis of this disease. In comparison, the second most frequent manifestation of TS is renal angiomyolipomata, detected in $67 \%$ of TS patients in this and other studies. ${ }^{10-13}$

The pathogenesis of pitted enamel hypoplasia in TS is not understood. ${ }^{4}$ Previous studies suggest that the pits extend to the amelodentinal junction. The pits appear to result from a reduction in the amount of enamel matrix formed. This may be because of a primary defect in odontoblasts, or in ameloblasts, or may be the result of defective interaction between odontoblasts and ameloblasts.

Dental enamel pits are not specific for TS. They are present in the general population although the exact prevalence is unclear. They are also associated with other abnormalities of amelogenesis, including pitted amelogenesis imperfecta, vitamin D dependent rickets, epidermolysis bullosa dystrophica, pseudohypoparathyroidism, and tricho-dento-osseous syndrome. ${ }^{6}$ These conditions are easily differentiated by the presence of additional dental defects such as pulp and root deformities, with the exception of pitted amelogenesis imperfecta. By contrast, the enamel defects in TS are mainly seen on the labial surfaces of the teeth away from the gingivae, in areas that rarely become carious. Water fluoridation may cause

Table 3 Previous studies looking at dental enamel pitting in tuberous sclerosis probands, relatives, and controls

\begin{tabular}{|c|c|c|c|c|c|c|}
\hline & \multicolumn{2}{|c|}{ Lygidakis and Lindenbaum ${ }^{6 *}$} & \multicolumn{2}{|c|}{ Mlynarczyk ${ }^{5} \dagger$} & \multicolumn{2}{|l|}{ Sampson $1992^{7} \ddagger$} \\
\hline & No & $\%$ with pits & No & $\%$ with pits & No & $\%$ with pits \\
\hline Probands & $\begin{array}{l}35 \text { typical TS } \\
10 \text { atypical TS }\end{array}$ & $\begin{array}{l}71 \\
10\end{array}$ & $\begin{array}{l}21<11 \mathrm{y} \\
29 \geqslant 11 \mathrm{y}\end{array}$ & $\begin{array}{l}76 \\
100\end{array}$ & $\begin{array}{l}6 \text { deciduous teeth } \\
23 \text { permanent } \\
\text { teeth }\end{array}$ & $\begin{array}{l}0 \\
48\end{array}$ \\
\hline Relatives & 68 & 13 & - & - & - & - \\
\hline Controls & 50 & 14 & 250 & 9 & 563 & $<1$ \\
\hline
\end{tabular}

*Used no dental stain and a fine probe.

†Used a dental plaque disclosing solution and a dental explorer.

$\ddagger$ Used no stain and a ball end probe. 
dental enamel pits, but the pits seen in our groups were unlike the typical lesions of fluorosis and were not associated with other features such as mottling.

In contrast to previous studies we found a high prevalence of pits in our controls ${ }^{5-7}$ (table 3 ). The variation in reported prevalence is probably related to differences in examination technique. Some studies did not use dental examining facilities, ${ }^{67}$ while it is unclear whether or not such facilities were used in two other studies. ${ }^{45}$ One study of TS cases and first degree relatives assessed pitting on clinical photographs of teeth. ${ }^{14}$ Dental probes vary in physical configuration. The CPITN probe used in one study is designed for examination of periodontal pockets rather than teeth. ${ }^{7}$ It has a $0.5 \mathrm{~mm}$ ball end which would not detect smaller pits and which would be less likely to "catch and keep" than a sharp dental explorer. The use of dental disclosing solutions increases the number of pits detected; only one previous investigator has used this technique. ${ }^{5}$ We feel that our higher rates of dental pitting are most likely the result of meticulous examination of the teeth being performed by a single dental hygienist using appropriate lighting, a dental plaque disclosing solution or stain, and a sharp dental explorer.

In the analysis of the dental enamel pit counts it is important to take the following factors into account. It is unlikely that analysis of pit size would be helpful in identifying the genetic status of unaffected relatives, as both large and small pits were seen in all three groups. The mean number of pits per tooth examined is not a useful measure nor is the total number of pits detected per person examined. Tooth type (for example, maxillary, central incisor, mandibular, canine, etc) is therefore a factor which may affect the number of pits present in any individual tooth. Any analysis which ignores this tooth type factor will be invalidated by differing numbers of each tooth type from person to person, given that not all the people examined will have all 12 anterior teeth present and unrestored. Statistical analysis using Poisson regression as used in this study allows incorporation of this tooth type factor, thus making better use of the data.

In summary, the prevalence of large numbers of pits in TS patients was striking $(70 \%$ probands had $>15$ pits and $84 \%$ of controls had $<6$ pits). This study does not answer the question as to whether asymptomatic first degree relatives with large numbers of pits have the TS genotype. Further studies of these subjects with high dental pit counts, when appropriate genetic testing becomes available, will determine their potential association with the TS phenotype.

We wish to thank Dr Alan Kelly from the Department of Community Health and General Practice, Trinity College Dublin for his assistance in the statistical analysis in this study.

1 European Chromosome 16 Tuberous Sclerosis Consortium 1993. Isolation and characterisation of the tuberous sclerosis gene on chromosome 16. Cell 1993;75:1305-15.

2 Webb DW, Osborne JP. Non-penetrance in tuberous sclerosis. F Med Genet 1991;28:417-19.

3 Al-Gazali LI, Arthur RJ, Lamb JT, et al. Diagnostic and counselling difficulties using a fully comprehensive screening protocol for families at risk for tuberous sclerosis. $\mathcal{f} \mathrm{Med}$ Genet 1989;26:694-703.

4 Hoff M, van Grunsven MF, Jongebloed WL, 's-Gravenmade EJ. Enamel defects associated with tuberous sclerosis. Oral Surg Oral Med Oral Pathol 1975;40:261-9.

5 Mlynarczyk G. Enamel pitting: a common symptom of tuberous sclerosis. Oral Surg Oral Med Oral Pathol 1991;71: 63-7.

6 Lygidakis NA, Lindenbaum RH. Pitted enamel hypoplasia in tuberous sclerosis patients and first degree relatives. Clin in tuberous sclerosis patie

7 Sampson JR, Attwood D, Al Mughery AS, Reid JS. Pitted enamel hypoplasia in tuberous sclerosis. Clin Genet 1992;42:50-2.

8 Weits-Binnerts JJ, Hoff $M$, van Grunsven NF. Dental pits in deciduous teeth, an early sign in tuberous sclerosis. Lancet 1982;ii:1344-5.

9 Roach E, Smith M, Huttenlocher P, Bhat M, Alcorn D, Hawley L. Diagnostic criteria: tuberous sclerosis complex. 7 Child Neurol 1992;7:221-4.

10 Golji $H$. Tuberous sclerosis and renal neoplasms. $f$ Urol 1961;85:919-23.

11 Lagos JC, Gomez MR. Tuberous sclerosis: reappraisal of a clinical entity. Mayo Clin Proc 1967;42:26-49.

2 Reed WB, Nickel WR, Campion G. Internal manifestation of tuberous sclerosis. Arch Dermatol 1963;87:715-28.

13 Wilson GC, Lo D. Tuberous sclerosis: a case with pulmonary and lymph node involvement. Med f Aust 1964; 2:795-6.

14 Fleury P, De Groot WP, Delleman JW, Verbeeten B Frankermolen-Witkiezwicz J. Tuberous sclerosis: the incidence of sporadic cases versus familial cases. Brain Dev 1980;2:107-17. 